Kragujevac Journal of Mathematics

Volume 46(1) (2022), Pages 21-28.

\title{
CONNECTEDNESS OF THE CUT SYSTEM COMPLEX ON NONORIENTABLE SURFACES
}

\author{
FATEMA ALI ${ }^{1,2}$ AND FERIHE ATALAN ${ }^{1}$
}

\begin{abstract}
Let $N$ be a compact, connected, nonorientable surface of genus $g$ with $n$ boundary components. In this note, we show that the cut system complex of $N$ is connected for $g<4$ and disconnected for $g \geq 4$. We then define a related complex and show that it is connected for $g \geq 4$.
\end{abstract}

\section{INTRODUCTION}

Complexes of curves and cut system complexes of surfaces are fundamental geometric objects in geometric topology. Let $S$ be a compact, connected, orientable or nonorientable surface of genus $g \geq 1$ with $n$ boundary components. Complexes of curves, denoted by $C(S)$, have been introduced by Harvey in $[3,4]$. Other geometric objects on surfaces include the cut system complex introduced by Hatcher and Thurston in [5]. They have played an ever increasing role since then.

In this note, we show that the cut system complexes of nonorientable surfaces are connected for $g<4$ and disconnected for $g \geq 4$. We then introduce a related complex and show that it is connected for $g \geq 4$.

\section{Preliminaries}

Let $a$ be a simple closed curve on $S$ and let $S_{a}$ denote the surface obtained by cutting $S$ along $a$. We call $a$ on the surface $S$ nonseparating if $S_{a}$ is connected, and separating otherwise. We denote a curve or its isotopy class by the same notation throughout this article. Let $\Sigma$ be a compact, connected, orientable surface of genus $g$ with $n$ boundary components. Let us consider collections of $g$ disjoint nonseparating

Key words and phrases. A nonorientable surface, cut system complex.

2010 Mathematics Subject Classification. Primary: 57N05. Secondary: 57M99, 05C40.

DOI 10.46793/KgJMat2201.021A

Received: January 11, 2019.

Accepted: August 03, 2019. 
simple closed curves $a_{1}, a_{2}, \ldots, a_{g}$ on $\Sigma$, whose complement $\Sigma \backslash\left(a_{1} \cup \cdots \cup a_{g}\right)$ is a sphere with $2 g+n$ boundary components. The collection of their isotopy classes is called a cut system on $\Sigma$. Let $\left\langle a_{1}, a_{2}, \ldots, a_{g}\right\rangle$ be a cut system on $\Sigma$. Assume that for some $k, a_{k}^{\prime}$ is a nonseparating simple closed curve transversely intersecting $a_{k}$ at exactly one point and disjoint from all $a_{i}$ for $i \neq k$. Then if we replace $a_{k}$ by $a_{k}^{\prime}$ in the cut system, we obtain another cut system on $\Sigma$. This operation of replacing curves is called an elementary move. There are three special types of paths which is described in [5]. These special types of paths play an important role in the construction of the cut system complex. The cut system complex of a surface $\Sigma$ is a cell complex of dimension 2. Each cut system is a 0-cell (vertex) of this complex. If two cells are related by an elementary move then these two 0-cells are joined by a 1-cell (an unoriented edge) corresponding to this move. Now, we have a graph, in other words; a 1-dimensional cell complex containing the 0-cells and the 1-cells. Finally, we attach 2-cells to this graph along the boundaries resulting from the three special types of paths to get the complex.

Hatcher and Thurston showed that the cut system complex of an orientable surface is connected in [5]. Later, Wajnryb proved the same result by elementary techniques in $[6]$.

An analogous complex for nonorientable surfaces seems to be the following. Let $N$ be a compact, connected nonorientable surface of genus $g$ with $n$ boundary components. If the regular neighborhood of the curve $a$ is a Möbius band or an annulus, then we say that $a$ is one-sided or two-sided, respectively. We note that all one-sided simple closed curves on $N$ are nonseparating. In addition, there are two topological types of one-sided simple closed curves on nonorientable surfaces of odd genus $g \geq 3$. Let $a$ be a one-sided simple closed curve. We call $a$ a one-sided essential simple closed curve if either $g=1$ or $g \geq 2$ and the surface $N_{a}$ is nonorientable. Otherwise, we say that $a$ is a one-sided characteristic simple closed curve. A cut system on the nonorientable surface $N$ is defined by taking a family of pairwise disjoint one-sided essential simple closed curves. Explicitly, let $\left\{a_{1}, a_{2}, \ldots, a_{g}\right\}$ be a collection of pairwise disjoint onesided essential simple closed curves on the surface $N$. Then, the collection of their isotopy classes $\left\langle a_{1}, a_{2}, \ldots, a_{g}\right\rangle$ is said to be a cut system if the surface obtained from $N$ by cutting along all $a_{i}$ in the collection is a sphere with $g+n$ boundary components. Let $\left\langle a_{1}, a_{2}, \ldots, a_{i-1}, a_{i}, a_{i+1}, \ldots, a_{g}\right\rangle$ be a cut system on the surface $N$. Let $a_{i}^{\prime}$ be a one-sided essential simple closed curve on the surface $N$ disjoint from $a_{k}$ for $k \neq i$, $1 \leq i \leq g$ and such that it intersects $a_{i}$ at one point and does not intersect other one-sided essential simple closed curves in the collection $\left\{a_{1}, a_{2}, \ldots, a_{g}\right\}$. Similar to the orientable case, if we change $a_{i}$ by $a_{i}^{\prime}$ in the collection, and we get a new cut system $\left\langle a_{1}, a_{2}, \ldots, a_{i-1}, a_{i}^{\prime}, a_{i+1}, \ldots, a_{g}\right\rangle$. This operation, introduced by Ashiba in [1], is called an elementary move. Also, the cut system complex, denoted by $\mathcal{O}(N)$, of a nonorientable surface is described in a similar fashion to the orientable case. As we will show in the next section, unfortunately this complex is not connected for the genus $g \geq 4$. 


\section{Main TheOREM}

In this section, firstly, we will explain below why $\mathcal{O}(N)$ is not connected for $g \geq 4$. Let $v_{1}=\left\langle d_{1}, a_{2}, \ldots, a_{g}\right\rangle$ and $v_{2}=\left\langle d_{2}, a_{2}, \ldots, a_{g}\right\rangle$ be vertices of the complex which are connected by an edge. Let $\bar{N}$ denote the surface $N$ whose holes are filled with discs. Since $d_{1}$ and $d_{2}$ intersect transversally once and they are disjoint from all $a_{i}$ 's we see that the homology classes of $d_{1}$ and $d_{2}$ are the same. This is because in the surface $\bar{N}$ both sums of the homology classes $\left[d_{1}\right]+\left[a_{2}\right]+\cdots+\left[a_{g}\right]$ and $\left[d_{2}\right]+\left[a_{2}\right]+\cdots+\left[a_{g}\right]$ are the Poincaré dual to the first Stiefel Whitney class so that $\left[d_{1}\right]+\left[a_{2}\right]+\cdots+\left[a_{g}\right]=\left[d_{2}\right]+\left[a_{2}\right]+\cdots+\left[a_{g}\right]$ and thus $\left[d_{1}\right]=\left[d_{2}\right]$. As a conclusion, we see that if two vertices of the complex are connected by an edge path then the homology classes of the isotopy classes in these vertices are pairwise identical in $\bar{N}$. In other words, if two vertices have a non-common homology class represented by the isotopy classes contained in them then these two vertices are not connected by an edge path. Such isotopy classes can be found for any $g \geq 4$, hence, the cut system complex cannot be connected in that case.

The above explanation raises the following question.

Question. What if we take $g-1$ pairwise disjoint one-sided essential simple closed curves $\left\{a_{1}, a_{2}, \ldots, a_{g-1}\right\}$ on $N$ as vertices, would the corresponding cut system complex made of these vertices be connected?

The answer is still negative. Indeed, if $v_{1}=\left\langle d_{1}, a_{2}, \ldots, a_{g-1}\right\rangle$ and $v_{2}=\left\langle d_{2}, a_{2}, \ldots, a_{g-1}\right\rangle$ are vertices of the complex, which are connected by an edge then cutting $N$ along all $a_{i}$ 's, we see that $d_{1}$ and $d_{2}$ are two one-sided curves inside a holed Klein Bottle intersecting transversally at one point. Hence again the homology classes $\left[d_{1}\right]$ and $\left[d_{2}\right]$ must coincide in $\bar{N}$. Therefore, the cut system complex still is not connected.

As a result of these observations, we have the following results.

Theorem 3.1. Let $N$ be a nonorientable surface of genus $g$ with $n$ holes, where $g<4$. Then the cut system complex $\mathcal{O}(N)$ is connected.

Let us take $g-2$ pairwise disjoint one-sided essential simple closed curves $\left\{a_{1}, a_{2}, \ldots, a_{g-2}\right\}$ on $N$ as vertices and let $X(N)$ denote the corresponding cut system complex made of these vertices. We call it partial cut system complex.

Theorem 3.2. Let $N$ be a nonorientable surface of genus $g$ with $n$ holes, where $g \geq 4$. Then the partial cut system complex $X(N)$ is connected.

The idea of the proofs is that any two vertices are connected by an edge path in the complex. We use Wajnryb's technique and follow his proof. The main ingredient used in the proof is the following proposition which is proved by Atalan and Korkmaz in $[2]$.

Proposition 3.1. Let $N$ be a nonorientable surface of genus $g$ with $n$ boundary components. Let $d_{1}$ and $d_{2}$ be two one-sided essential simple closed curves on the 
surface $N$ such that $i\left(d_{1}, d_{2}\right)=k$, where $k \geq 2$. In this case, there is a one-sided essential simple closed curve $d$ such that $i\left(d, d_{1}\right)<k$ and $i\left(d, d_{2}\right)<k$.

Proof of Theorem 3.1. Let $g=1$. In this case, a cut system (on the surface $N$ ) contains an isotopy class of a single curve. If two distinct one-sided curves intersect at one point, we connect them by an edge. Since any two essential one-sided curves on a genus one nonorientable surface intersect, using induction it follows from Proposition 3.1 that any two one-sided essential curves can be joined by an edge path in the cut system complex $\mathcal{O}(N)$.

Let $g=2$. Let $v_{1}$ and $v_{2}$ be any two vertices of the complex $\mathcal{O}(N)$. We will show that there exists an edge path $P=\left(v_{1}=s_{1}, s_{2}, \ldots, s_{k}=v_{2}\right)$ connecting $v_{1}$ and $v_{2}$. There are two cases.

Case 1. Suppose that the vertices $v_{1}$ and $v_{2}$ have one isotopy class of one-sided essential simple closed curve in common, say $d$. Let us cut the surface $N$ along the curve $d$. The collection of the remaining one-sided essential simple closed curves constitute two vertices of the cut system complex on the obtained surface of genus one. We have showed that the complex $\mathcal{O}(N)$ is connected for $g=1$. So, they can be connected by a path. Including this common curve $d$ to each of the vertices of this path we obtain a path in $\mathcal{O}(N)$ connecting $v_{1}$ to $v_{2}$.

Case 2. Suppose that the vertices $v_{1}$ and $v_{2}$ do not have any common isotopy class of one-sided essential simple closed curves. Let $d_{1}$ and $d_{2}$ be two different isotopy classes of one-sided essential simple closed curves on $N$ such that $v_{1}$ and $v_{2}$ contain $d_{1}$ and $d_{2}$, respectively. Then, we need to show that there exists an edge path connecting $v_{1}$ and $v_{2}$. To prove this, as in the proof of Lemma 17 in [6], we will use induction on $i\left(d_{1}, d_{2}\right)=n$.

There are three subcases.

Subcase (i). Let $i\left(d_{1}, d_{2}\right)=0$. Then there is a vertex $u$ containing both one-sided essential curves $d_{1}$ and $d_{2}$. Hence, the vertex $u$ is connected to $v_{1}$ and $v_{2}$ as in Case 1 .

Subcase (ii). Let $i\left(d_{1}, d_{2}\right)=1$. The regular neighborhood of $d_{1} \cup d_{2}$ is a two-holed real projective plane. Let us denote $N_{d_{1} \cup d_{2}}$ the surface obtained by cutting $N$ along $d_{1}$ and $d_{2}$. Since $g=2, N_{d_{1} \cup d_{2}}$ has necessarily two components, one of which is a nonorientable surface of genus one, so that we can find a one-sided essential simple closed curve disjoint from $d_{1}$ and $d_{2}$, say $e$. Now, we can find two vertices $w_{1}$ and $w_{2}$ in the complex $\mathcal{O}(N)$ which are joined by an edge and such that $w_{1}$ and $w_{2}$ contain $d_{1}$ and $d_{2}$, respectively. In other words, $w_{1}=\left\langle d_{1}, e\right\rangle$ and $w_{2}=\left\langle d_{2}, e\right\rangle$ are connected by an edge. Finally, we join $v_{1}$ to $w_{1}$ and $v_{2}$ to $w_{2}$ as in Case 1 . Therefore, we can connect $v_{1}$ and $v_{2}$.

Subcase (iii). Let $i\left(d_{1}, d_{2}\right)=n>1$. By Proposition 3.1, there is a one-sided essential simple closed curve $d$ such that $i\left(d_{1}, d\right)<n$ and $i\left(d_{2}, d\right)<n$. We choose a vertex $u$ containing $d$. By induction on $n$, we can connect the vertex $u$ to $v_{1}$ and $v_{2}$ in the cut system complex $\mathcal{O}(N)$. 
For the case $g=3$ the proof is the same as for the case $g=2$ except the Subcase (ii), which we include below.

Subcase (ii). Let $i\left(d_{1}, d_{2}\right)=1$. Since $g=3$ and $d_{1}$ and $d_{2}$ are both essential, $N_{d_{1} \cup d_{2}}$ has necessarily two components. Moreover, either both components are nonorientable of genus one or one of the components is a nonorientable surface of genus two and the other is a sphere with holes. Hence, in each case, we can find two disjoint onesided essential simple closed curves $e_{1}$ and $e_{2}$ that they are both disjoint from $d_{1}$ and $d_{2}$. Now, we can find two vertices $w_{1}$ and $w_{2}$ in the complex which are joined by an edge and such that $w_{1}$ and $w_{2}$ contain $d_{1}$ and $d_{2}$, respectively. In other words, $w_{1}=\left\langle d_{1}, e_{1}, e_{2}\right\rangle$ and $w_{2}=\left\langle d_{2}, e_{1}, e_{2}\right\rangle$ are connected by an edge. Finally, we join $w_{1}$ to $v_{1}$ and $v_{2}$ to $w_{2}$ as in Case 1 . Therefore, we can connect $v_{1}$ and $v_{2}$.

This completes the proof of the theorem.

Proof of Theorem 3.2. We use induction on the genus of the surface $N$ for $g \geq 4$.

Let $g=4$. As in the above proof, there are two cases.

Case 1. Assume that $v_{1}$ and $v_{2}$ have one isotopy class of one-sided essential curve in common, say $d$. Let $e$ and $f$ be the other one-sided essential simple closed curves of $v_{1}$ and $v_{2}$, respectively. In other words, let $v_{1}=\langle d, e\rangle$ and $v_{2}=\langle d, f\rangle$. We will show that $v_{1}$ is connected to $v_{2}$ by an edge path. In this case, there are three possibilities.

- The one-sided essential curves $e$ and $f$ are disjoint. Then, since $d$ is disjoint from both $e$ and $f$, and these all curves are one-sided essential, there is another essential simple closed curve, say $g$, in the complement of $d \cup e \cup f$. Hence, we can find an essential one-sided simple closed curve $c$ representing $\mathbb{Z}_{2}$-homology class $[e]+[f]+[g]$ such that $c$ intersects each of $e$ and $f$ at only one point. Thus, we obtain the required path.

- The one-sided essential curves $e$ and $f$ intersect at one point. Then there is nothing to prove.

- The one-sided essential curves $e$ and $f$ intersect at least two points. Let us cut the surface $N$ along the curve $d$. We get a nonorientable surface of genus three, say $N_{d}$, in which $i(e, f)=k \geq 2$. First assume that the curves $e$ and $f$ are still essential in $N_{d}$. Then, by Proposition 3.1, there is a one-sided essential simple closed curve $c$ such that $i(e, c)<k$ and $i(f, c)<k$.

Now assume without loss of generality that the curve $e$ is not essential in $N_{d}$. Hence $e$ is characteristic in $N_{d}$. Since $f$ is one-sided and $e$ is characteristic the integer $k$ must be odd. Take representatives for $e$ and $f$ and a push-off of $f$ that intersects $f$ transversally at one point. Also a take a slight perturbation $f^{\prime}$ of the push-off. By inspection we see that our curves must be as in the diagram at Figure 1.

This new curve $f^{\prime}$ has the same homology class as $f$. It has at most two components. If it is connected then it, call it $c$, intersects $f$ in one point and $e$ in $k-2$ points. Since $k-2$ is odd, $c$ is still one-sided. If the $f^{\prime}$ is not connected, then by homology arguments one of the components must be one-sided and the other one must be two-sided. The one-sided component, call it $c$ again, will intersect $f$ in at most one point and $e$ in at 


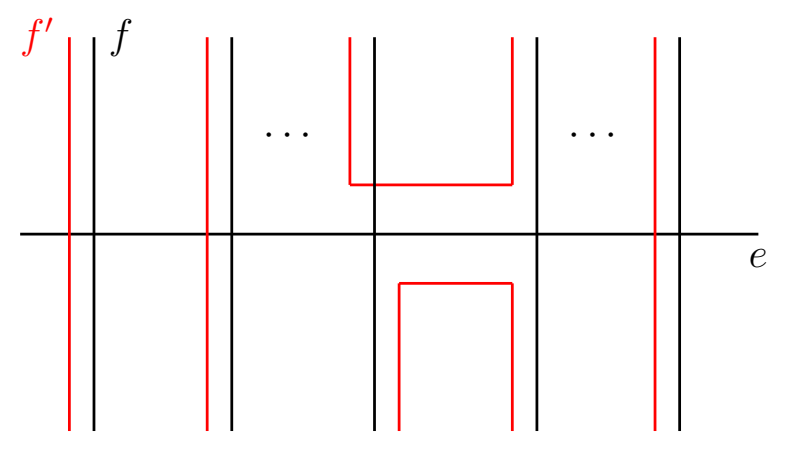

FIGURE 1.

most $k-2$ points. The curve $c$ may not be essential in $N_{d}$ but certainly is essential in $N$.

In particular, in all cases we have obtained a one-sided essential simple closed curve $c$ such that $i(e, c)<k$ and $i(f, c)<k$. Finally, using an induction argument we obtain two sequences of essential one-sided simple closed curves $e=e_{0}, e_{1}, \ldots, e_{j}=c$ and $c=c_{0}, c_{1}, \ldots, c_{s}=f$ such that any two adjacent essential one-sided simple closed curves in the sequences intersect once. Then the sequence $e=e_{0}, e_{1}, \ldots, e_{j}=c=$ $c_{0}, c_{1}, \ldots, c_{s}=f$ gives the required path in $X(N)$.

Case 2. Assume that the vertices $v_{1}$ and $v_{2}$ do not have any common isotopy class of one-sided essential simple closed curves. Let $d_{1}$ and $d_{2}$ be two different isotopy classes of one-sided essential simple closed curves on $N$ such that $v_{1}$ and $v_{2}$ contain $d_{1}$ and $d_{2}$, respectively. Then, we show that there exists an edge path connecting $v_{1}$ and $v_{2}$. To prove this, as in the proof of Lemma 17 in [6], we will use induction on $i\left(d_{1}, d_{2}\right)=n$.

There are three subcases.

Subcase (i). Let $i\left(d_{1}, d_{2}\right)=0$. Then, we can construct a vertex $u=\left\langle d_{1}, d_{2}\right\rangle$. Thus, $u$ is connected to $v_{1}$ and $v_{2}$ as in Case 1 .

Subcase (ii). Let $i\left(d_{1}, d_{2}\right)=1$. The regular neighborhood of $d_{1} \cup d_{2}$ is a two-holed real projective plane. Then, there are two possibilities. One possibility is that $N_{d_{1} \cup d_{2}}$ is a connected nonorientable surface of genus 1 (note that $N_{d_{1} \cup d_{2}}$ cannot be connected and orientable since both $d_{1}$ and $d_{2}$ are essential). Hence, we can find a one-sided essential simple closed curve disjoint from $d_{1}$ and $d_{2}$. Again we can find two vertices $w_{1}$ and $w_{2}$ containing $d_{1}$ and $d_{2}$, respectively, such that they are connected by an edge. Hence, we join $w_{1}$ to $v_{1}$ and $v_{2}$ to $w_{2}$ as in Case 1. The other possibility is that $N_{d_{1} \cup d_{2}}$ is disconnected. We can notice that at least one component of $N_{d_{1} \cup d_{2}}$ must be nonorientable, so in this case we can also find a one-sided essential simple closed 
curve disjoint from $d_{1}$ and $d_{2}$, which allows to treat the disconnected case similarly. Therefore, we can connect $v_{1}$ and $v_{2}$ by a path.

Subcase (iii). Let $i\left(d_{1}, d_{2}\right)=n>1$. By Proposition 3.1, there is a one-sided essential simple closed curve $d$ such that $i\left(d_{1}, d\right)<n$ and $i\left(d_{2}, d\right)<n$. Let us pick a vertex $u$ containing $d$. By induction on $n$, we can join $u$ to $v_{1}$ and $v_{2}$.

Let $g \geq 5$. By the induction hypothesis, we assume that the theorem holds for a nonorientable surface of genus less than $g$. We will prove that the complex $X(N)$ is connected for a nonorientable surface of genus $g$. Let $v_{1}$ and $v_{2}$ be any two vertices of the complex $X(N)$. We will prove that these two vertices are connected by an edge path.

Case 1. Suppose that $v_{1}$ and $v_{2}$ have one isotopy class of one-sided essential curve in common, say $d$. Let us cut the surface $N$ along the curve $d$. The collection of the remaining one-sided essential simple closed curves constitute two vertices of the cut system complex on the obtained surface of smaller genus. By the induction hypothesis, they can be connected by a path. Including this common curve $d$ to each of the vertices of this path we obtain a path in $X(N)$ connecting $v_{1}$ to $v_{2}$.

Case 2. Suppose that $v_{1}$ and $v_{2}$ do not have any common isotopy classes of onesided essential simple closed curves. Let $d_{1}$ and $d_{2}$ be two different isotopy classes of one-sided essential simple closed curves on $N$ such that $v_{1}$ and $v_{2}$ contain $d_{1}$ and $d_{2}$, respectively. To prove the existence of an edge path joining $v_{1}$ and $v_{2}$, as in the proof of Lemma 17 in [6], we will use induction on $i\left(d_{1}, d_{2}\right)=n$.

There are three subcases.

Subcase (i). Let $i\left(d_{1}, d_{2}\right)=1$. The regular neighborhood of $d_{1} \cup d_{2}$ is a two-holed real projective plane. Again, we have two possibilities. One possibility is that $N_{d_{1} \cup d_{2}}$ is a connected nonorientable surface of genus $g-3$. So, one can choose pairwise disjoint $g-3$ one-sided essential curves disjoint from $d_{1}$ and $d_{2}$. Then, there are vertices $w_{1}$ and $w_{2}$ containing $d_{1}$ and $d_{2}$, respectively, such that they are joined by an edge. Thus, we connect $w_{1}$ to $v_{1}$ and $v_{2}$ to $w_{2}$ as in Case 1 . The other possibility is that $N_{d_{1} \cup d_{2}}$ is disconnected. However, by Theorem 3.10 in [2], we can find a sequence of essential one-sided simple closed curves $d_{1}=a_{1}, a_{2}, \ldots, a_{k}=d_{2}$ such that any two adjacent curves $a_{i}$ and $a_{i+1}$ in the sequence intersect once and $N_{a_{i} \cup a_{i+1}}$ is connected, where $N_{a_{i} \cup a_{i+1}}$ is the surface obtained by cutting $N$ along $a_{i}$ and $a_{i+1}$. Therefore, using the idea of the previous possibility, we can connect $v_{1}$ and $v_{2}$ by a path.

Subcase (ii). Let $i\left(d_{1}, d_{2}\right)=0$. If $\left[d_{1}\right]+\left[d_{2}\right]$ is not characteristic, then there is a vertex $u$ containing both curves $d_{1}$ and $d_{2}$. Hence, the vertex $u$ is connected to $v_{1}$ and $v_{2}$ as in Case 1 . Now, assume that $\left[d_{1}\right]+\left[d_{2}\right]$ is characteristic, in this case $g \geq 6$. Then, $\left[d_{2}\right]$ is characteristic on $N_{d_{1}}$ which is a connected nonorientable surface of genus $g \geq 5$. Without lost of generality, we can choose a one-sided essential curve $c$ on $N_{d_{1}}$, which is not characteristic such that $i\left(c, d_{2}\right)=1$. This implies that $[c]+\left[d_{1}\right]$ is not characteristic on $N$. So there is a vertex $u$ containing both curves $d_{1}$ and $c$. Now, we can connect the vertex $u$ and $v_{1}$ as in Case 1 . Moreover, $u$ can be connected to $v_{2}$ because $i\left(c, d_{2}\right)=1$ by Subcase (i) above. 
Subcase (iii). Let $i\left(d_{1}, d_{2}\right)=n>1$. By Proposition 3.1, there is a one-sided essential simple closed curve $d$ such that $i\left(d_{1}, d\right)<n$ and $i\left(d_{2}, d\right)<n$. We pick a vertex $u$ containing $d$. By induction on $n$, we can connect the vertex $u$ to $v_{1}$ and $v_{2}$ in the partial complex $X(N)$.

This finishes the proof of the theorem.

Acknowledgements. The authors would like to thank the referee for his/her valuable corrections and suggestions.

\section{REFERENCES}

[1] S. A. S. Ashiba, The Hatcher-Thurston complex on a surface, Ms. Thesis, Atilim University, Ankara, 2016.

[2] F. Atalan and M. Korkmaz, Automorphisms of curve complexes on nonorientable surfaces, Groups Geom. Dyn. 8 (2014), 39-68.

[3] W. J. Harvey, Geometric structure of surface mapping class groups, in: C. T. C. Wall (Ed.), Homological Group Theory, London Mathematical Soc. Lecture Notes Series 36, Cambridge University Press, Cambridge, 1979, 255-269.

[4] W. J. Harvey, Boundary structure of the modular group, in: I. Kra and B. Maskit (Eds.), Riemann Surfaces and Related Topics: Proceedings of the 1978 Stony Brook Conference, Annals of Math. Studies 97, Princeton University Press, Princeton, New Jersey, 1981, 245-251.

[5] A. Hatcher and W. Thurston, A presentation of the mapping class group of a closed orientable surface, Topology 19 (1980), 221-237.

[6] B. Wajnryb, An elementary approach to the mapping class group of a surface, Geometry and Topology 3 (1999), 405-466.

${ }^{1}$ Department of Mathematics, Atilim University, 06830 AnkARA, TURKey

Email address: ali.fatema@student.atilim.edu.tr

Email address: ferihe.atalan@atilim.edu.tr

${ }^{2}$ Department of Mathematics, BANI WALIED UNIVERSITY, LIBYA 